\title{
Asymptotic properties of spectral estimates of second order
}

\author{
BY DAVID R. BRILLINGER \\ London School of Economics and Political Science
}

\section{SUMMARY}

Let $\mathbf{X}(t)(t=0, \pm 1, \ldots)$ be a zero mean, $r$ vector-valued, strictly stationary time series satisfying a particular assumption about the near-independence of widely separated values. Given the values $\mathbf{X}(t)(t=0,1, \ldots, T-1)$, we construct the statistics: $\mathbf{I}_{X}^{\left(T^{\prime}\right)}(\lambda)(-\infty<\lambda<\infty)$, the matrix of second-order periodograms, $\mathbf{F}_{X X}^{\left(T^{\prime}\right)}(\lambda)$, the matrix of sample spectral measures, $\mathbf{f}_{X X}^{\left(T^{\prime}\right)}(\lambda)$, the matrix of sample spectral densities and $\mathbf{c}_{X X}^{(T)}(u)(u=0, \pm 1, \ldots)$, the matrix of sample covariances. In the paper expressions are derived for the first- and second-order moments and the asymptotic distributions of $\mathbf{I}_{X X}^{\left(T_{X}^{\prime}\right)}(\lambda), \mathbf{F}_{X X}^{(T)}(\lambda), \mathbf{f}_{X X}^{(T)}(\lambda)$ and $\mathbf{c}_{X X}^{\left(T^{\prime}\right)}(u)$. Our purpose is to determine the form of these moments and to indicate the appearance of the Wishart distribution as an exact limiting distribution for $f_{X X}^{(T)}(\lambda)$. It has previously been suggested as an approximation.

\section{IntroduCtion}

We consider asymptotic properties of second-order statistics based on sample values from a strictly stationary vector-valued time series. The series is assumed to possess moments of all orders and to be such that values of the series, well separated in time, are nearly stochastically independent. This weak span of dependence requirement is formulated as Assumption I. It is a principal and unifying assumption of the theorems presented.

The statistics considered are based on the matrix of second-order periodograms. Our method of proceeding is to derive a general theorem on the asymptotic behaviour of the periodogram, including a necessary uniform error term, and then to deduce the behaviour of the other statistics from this. In fact, the periodograms are based on the discrete Fourier transform of the sample. A lemma of Brillinger \& Rosenblatt (1967) indicates the elementary asymptotic sampling properties of this transform. The work of Tukey (1967) indicates the extreme rapidity with which it may be calculated and the consequent quick calculation of the statistics of this paper. In addition the pleasant analytic properties of Fourier transforms are well known. We have therefore been led to take the periodogram as the basis of our work for three distinct and important reasons. Our work differs from much of the previous work in giving the periodogram such an important position. A further important distinction from previous work is that no assumption about the linearity of the underlying process is required for the results presented here.

We prove that distinct values of the periodogram tend to be asymptotically independent and have Wishart distributions. The sample spectral measure, $\mathbf{F}_{X X}^{(T)}(\lambda)$, tends to be Gaussian with a spectrum of order four, a trispectrum, appearing in its distribution. The sample autocovariance function, $\mathbf{c}_{X X}^{(T)}(u)$, is also seen to be asymptotically Gaussian, the distribution again involving a trispectrum. We demonstrate the convergence of these statistics, considered as random functions of $\lambda$ and $u$ respectively, to limiting Gaussian processes.

Two limiting distributions are seen to appear in the case of the sample spectral density 
matrix. Under one limiting process it tends to be Gaussian and under a second it tends to have a Wishart distribution.

We commence to set down notation. Let $\mathbf{X}(t)(t=0, \pm 1, \ldots)$ be a strictly stationary $r$ vector-valued time series all of whose moments exist. Set

$$
\begin{aligned}
E\{\mathbf{X}(t)\} & =\mathbf{c}_{X}, \\
E\left[\left\{\mathbf{X}(t+u)-\mathbf{c}_{X}\right\}\left\{\mathbf{X}(t)-\mathbf{c}_{X}\right\}^{\prime}\right] & =\mathbf{c}_{X X}(u) \quad(t, u=0, \pm 1, \ldots) .
\end{aligned}
$$

Suppose

$$
\sum_{u=-\infty}^{\infty}\left|\mathbf{c}_{X X}(u)\right|<\infty
$$

Here $\left|\mathbf{c}_{X X}(u)\right|$ denotes the matrix of absolute values. We may then define $\mathbf{f}_{X X}(\lambda)$, the $r \times r$ matrix of second-order spectral densities, by

$$
\mathbf{f}_{X X}(\lambda)=(2 \pi)^{-1} \sum_{u=-\infty}^{\infty} \mathbf{c}_{X X}(u) \exp (-i \lambda u) \quad(-\infty<\lambda<\infty)
$$

and $\mathbf{F}_{X X}(\lambda)$, the matrix of second-order spectral measures, by

$$
\mathbf{F}_{X X}(\lambda)=\int_{0}^{\lambda} \mathbf{f}_{X X}(\alpha) d \alpha \quad(0 \leqslant \lambda \leqslant \pi) .
$$

We suppose that $\mathbf{X}(t)$ has a weak span of time dependence as indicated by Assumption $\mathbf{I}$.

We construct estimates $\mathbf{c}_{X X}^{(T)}(u), \mathbf{f}_{X X}^{(T)}(\lambda)$ and $\mathbf{F}_{X X}^{(T)}(\lambda)$ of $\mathbf{c}_{X X}(u), \mathbf{f}_{X X}(\lambda)$ and $\mathbf{F}_{X X}(\lambda)$. These estimates are based on $\mathbf{I}_{X X}^{(T)}(\lambda)$, the matrix of second-order periodograms. This last is derived from the finite Fourier transform of an observed stretch of data, $\mathbf{X}(t)(t=0,1, \ldots, T-1)$.

We determine asymptotic expressions for the cumulants of $\mathbf{c}_{X X}^{\left(T_{X}^{\prime}\right)}(u), \mathbf{I}_{X X}^{\left(T_{X}\right)}(\lambda), \mathbf{F}_{X X}^{\left(T_{X}\right)}(\lambda)$ and $\mathbf{f}_{X X}^{(T)}(\lambda)$ and from these cumulants are able to identify the limiting distributions of the appropriately standardized estimates. We also consider the weak convergence of the sequences of stochastic processes

$$
\left\{\mathbf{c}_{X X}^{\left(T^{\prime}\right)}(u)(u=0, \pm 1, \ldots)\right\}, \quad\left\{\mathbf{F}_{X X}^{\left(T^{\prime}\right)}(\lambda)(0 \leqslant \lambda \leqslant \pi)\right\} \quad \text { and } \quad\left\{\mathbf{f}_{X X}^{\left(T^{\prime}\right)}(\lambda)(-\infty<\lambda<\infty)\right\} .
$$

We do not assume that $\mathbf{X}(t)$ is a linear process.

In the paper $W_{r}(\nu, \boldsymbol{\Sigma})$ will denote an $r \times r$ symmetric matrix-valued Wishart variate with variance-covariance matrix $\boldsymbol{\Sigma}$ and $\nu$ degrees of freedom. Let $W_{r}^{C}(\nu, \boldsymbol{\Sigma})$ denote an $r \times r$ Hermitian matrix-valued complex Wishart variate with variance-covariance matrix $\boldsymbol{\Sigma}$ and $\nu$ degrees of freedom. This last distribution is discussed by Goodman (1963). For real matrices $\mathbf{A}, \mathbf{B}$ and $\mathbf{Z}=\mathbf{A}+i \mathbf{B}$, write

$$
\mathbf{Z}^{R}=\left[\begin{array}{rr}
\mathbf{A} & \mathbf{B} \\
-\mathbf{B} & \mathbf{A}
\end{array}\right]
$$

then the two are connected by $W_{r}^{C}(\nu, \boldsymbol{\Sigma})^{R}=W_{2 r}\left(\nu, \boldsymbol{\Sigma}^{R}\right)$. We set

$$
\begin{gathered}
\Delta^{(T)}(\lambda)=\sum_{t=0}^{T-1} \exp (-i \lambda t), \\
\eta(\lambda)=\left\{\begin{array}{ll}
1 & (\lambda \equiv 0, \bmod 2 \pi), \\
0 & \text { otherwise }
\end{array}\right\}
\end{gathered}
$$

For $\left(Y_{1}, Y_{2}, \ldots, Y_{k}\right)$ a random variable with real or complex components, we denote its joint cumulant of order $k$ by

$$
\operatorname{cum}\left(Y_{1}, Y_{2}, \ldots, Y_{k}\right)
$$

This is the coefficient of $t_{1} t_{2} \ldots t_{k}$ in the expansion of its cumulant generating function. For $X, Y$ complex-valued, $\operatorname{cov}(X, Y)=E\left[\left\{(X-E(X)\}\{\bar{Y}-E(\bar{Y})\}^{\prime}\right]\right.$. 


\section{Parameters and estimates}

Let the $r$ vector-valued series $\mathbf{X}(t)$ have real-valued components $X_{a}(t)(a=1,2, \ldots, r)$. All moments are assumed to exist and we set

$$
\begin{aligned}
c_{a_{1}, \ldots, a_{k}}\left(t_{1}, \ldots, t_{k-1}\right) & =\operatorname{cum}\left\{X_{a_{1}}\left(t_{1}+\tau\right), \ldots, X_{a_{k-1}}\left(t_{k-1}+\tau\right), X_{a_{k}}(\tau)\right\} \\
& \left(a_{1}, \ldots, a_{k}=1,2, \ldots, r ; t_{1}, \ldots, t_{k-1}, \tau=0, \pm 1, \ldots ; k=1,2, \ldots\right)
\end{aligned}
$$

using the assumed stationarity. We then set down

Assumption I. $\mathbf{X}(t)$ is a strictly stationary series all of whose moments exist. For each $j=1,2, \ldots, k-1$ and any $k$-tuple $a_{1}, a_{2}, \ldots, a_{k}$ we have

$$
\sum_{t_{1}, \ldots, t_{k-1}}\left|t_{j} c_{a_{1}, \ldots, a_{k-1}}\left(t_{1}, \ldots, t_{k-1}\right)\right|<\infty \quad(k=2,3, \ldots) .
$$

Because cumulants are measures of the joint dependence of random variables, $(2 \cdot 2)$ is seen to be a form of mixing or asymptotic independence requirement for values of $\mathbf{X}(t)$ well separated in time. In the case of a Gaussian series, because cumulants of order greater than 2 vanish, Assumption $I$ is satisfied if one requires only

$$
\sum_{t=-\infty}^{\infty}\left|t c_{a a}(t)\right|<\infty
$$

where $c_{a a}(t)$ is the autocovariance function of $X_{a}(t)(a=1,2, \ldots, r)$.

If $\mathbf{X}(t)$ satisfies Assumption I we may define its cumulant spectral densities by

$$
\begin{array}{r}
f_{a_{1} \ldots, a_{k}}\left(\lambda_{1}, \ldots, \lambda_{k-1}\right)=(2 \pi)^{-k+1} \sum_{t_{1}, \ldots, t_{k-1}} c_{a_{1}, \ldots, a_{k}}\left(t_{1}, \ldots, t_{k-1}\right) \exp \left(-i \sum_{j=1}^{k-1} \lambda_{j} t_{j}\right) \\
\left(-\infty<\lambda<\infty ; a_{1}, \ldots, a_{k}=1,2, \ldots, r ; k=1,2, \ldots\right) .
\end{array}
$$

If $k=2$, the cross-spectra $f_{a_{1} a_{2}}(\lambda)$ are collected together in the matrix $\mathbf{f}_{X X}(\lambda)$ of $(1 \cdot 4)$.

Suppose now that a stretch, $\mathbf{X}(t)(t=0,1, \ldots, T-1)$ of the series $\mathbf{X}(t)$ is available. For $-\infty<\lambda<\infty$, we define

$$
\mathbf{d}_{X}^{(T)}(\lambda)=\sum_{t=0}^{T-1} \exp (-i \lambda t) \mathbf{X}(t)
$$

the finite Fourier transform of the given stretch of data. Denote the entries of $\mathbf{d}_{X}^{(T)}(\lambda)$ by $d_{a}^{(T)}(\lambda)(a=1,2, \ldots, r)$. Following Brillinger \& Rosenblatt (1967) one has

Lemma 2-1. Suppose Assumption I is satisfied, then

$$
\operatorname{cum}\left\{d_{a_{1}}^{(T)}\left(\lambda_{1}\right), \ldots, d_{a_{1}}^{(T)}\left(\lambda_{k}\right)\right\}=(2 \pi)^{k-1} f_{a_{1}, \ldots, a_{k}}\left(\lambda_{1}, \ldots, \lambda_{k-1}\right) \Delta^{(T)}\left(\sum_{j=1}^{k} \lambda_{j}\right)+O(1) .
$$

The error term $O(1)$ is uniform in $\lambda_{1}, \ldots, \lambda_{k}$ as $T \rightarrow \infty$.

Suppose that $E\{\mathbf{X}(t)\}=0$; then this lemma indicates that one might base estimates of $\mathbf{f}_{X X}(\lambda)$ upon

$$
\mathbf{I}_{X X}^{(T)}(\lambda)=(2 \pi T)^{-1} \mathbf{d}^{(T)}(\lambda) \overline{\mathbf{d}}^{(T)^{\prime}}(\lambda)
$$

the matrix of second-order periodograms; the bar denotes complex conjugate. As an estimate of $\mathbf{F}_{X X}(\lambda)$ we consider

$$
\mathbf{F}_{X X}^{(T)}(\lambda)=\int_{0}^{\lambda} \mathbf{I}_{X X}^{(T)}(\alpha) d \alpha \quad(0 \leqslant \lambda \leqslant \pi)
$$


As an estimate of $\mathbf{c}_{X X}(u)$, in this case where $E\{\mathbf{X}(t)\}=0$, we consider

$$
\begin{aligned}
\mathbf{m}_{X X}^{\left(T^{\prime}\right)}(u) & =\sum_{0 \leqslant t, t+u \leqslant T-1} \mathbf{X}(t+u) \mathbf{X}^{\prime}(t) \\
& =\int_{-\pi}^{\pi} \mathbf{I}_{X X}^{(T)}(\alpha) \exp (i u \alpha) d \alpha .
\end{aligned}
$$

Before constructing an estimate of $\mathbf{f}_{X X}(\lambda)$, we set down

Assumptron II. Let $H(\alpha)(-\pi<\alpha \leqslant \pi)$ be a weight function that is bounded, is symmetric about 0 , has a bounded first derivative and is such that

Given $B_{T}>0$, we then set

$$
\int_{-\pi}^{\pi} H(\alpha) d \alpha=1
$$

$$
H^{(T)}(\alpha)=B_{T}^{-1} H\left(B_{T}^{-1} \alpha\right) .
$$

In later sections we will consider the cases: $B_{T}=K / T ; B_{T} \rightarrow 0, B_{T} T \rightarrow \infty$ as $T \rightarrow \infty$; $B_{T}$ constant with respect to $T$.

As an estimate of $\mathbf{f}_{X X}(\lambda)$, we take

$$
\begin{aligned}
\mathbf{f}_{X X}^{(T)}(\lambda) & =\int_{-\pi}^{\pi} H^{(T)}(\alpha) \mathbf{I}_{X X}^{(T)}(\lambda-\alpha) d \alpha \\
& =(2 \pi)^{-1} \sum_{u=-T+1}^{T-1} \mathbf{m}_{X X}^{(T)}(u) \exp (-i u \lambda) \int_{-\pi}^{\pi} H^{(T)}(\alpha) \exp (-i u \alpha) d \alpha .
\end{aligned}
$$

We have been led to consider a variety of statistics based on $\mathbf{I}_{X X}^{(T)}(\lambda)$, the matrix of secondorder periodograms, and therefore turn to an investigation of its asymptotic properties.

We note that Bartlett (1966, p. 337) has suggested handling the sampling theory of vector-valued series $\mathbf{X}(t)$ by means of arbitrary linear combinations $\alpha^{\prime} \mathbf{X}(t)$, with $\alpha$ an $r$ vector.

\section{The PERIODOgRAM}

Because all of the moments of $\mathbf{X}(t)$ are finite, all of the moments of $\mathbf{I}_{X X}^{(T)}(\lambda)$ will be finite. We turn to a determination of the asymptotic cumulants of $I_{X X}^{(T)}(\lambda)$. We do this by using the rules developed by Leonov \& Shiryaev (1959) for determining the joint cumulants of polynomial functions of random variables.

Denote the entry in the $a$ th row and $b$ th column of $\mathbf{I}_{X X}^{(T)}(\lambda)$ by $I_{a b}^{(T)}(\lambda)(a, b=1,2, \ldots, r)$. We then have

Theorem 3·1. Let $\mathbf{X}(t)$ satisfy Assumption I and have mean 0. Then

with $O\left(T^{-1}\right)$ uniform in $\lambda_{1}$,

$$
E\left\{I_{a_{1} b_{1}}^{(T)}\left(\lambda_{1}\right)\right\}=f_{a_{1} b_{1}}\left(\lambda_{1}\right)+O\left(T^{-1}\right)
$$

$$
\begin{aligned}
\operatorname{cov}\left\{I_{a_{1} b_{1}}^{(T)}\left(\lambda_{1}\right), I_{a_{2} b_{2}}^{(T)}\left(\lambda_{2}\right)\right\}=T^{-2} & \left|\Delta^{(T)}\left(\lambda_{1}-\lambda_{2}\right)\right|^{2} f_{a_{1} a_{2}}\left(\lambda_{1}\right) f_{b_{1} b_{2}}\left(-\lambda_{1}\right) \\
& +T^{-2}\left|\Delta^{(T)}\left(\lambda_{1}+\lambda_{2}\right)\right|^{2} f_{a_{1} b_{2}}\left(\lambda_{1}\right) f_{b_{1} a_{2}}\left(-\lambda_{1}\right) \\
& +2 \pi T^{-1} f_{a_{1} b_{1} a_{2} b_{2}}\left(\lambda_{1},-\lambda_{1},-\lambda_{2}\right)+T^{-2} R^{(T)}\left(\lambda_{1}, \lambda_{2}\right)
\end{aligned}
$$

where there is a finite $K$ such that

$$
\left|R^{(T)}\left(\lambda_{1}, \lambda_{2}\right)\right| \leqslant K\left\{\left|\Delta^{(T)}\left(\lambda_{1}+\lambda_{2}\right)\right|+\left|\Delta^{(T)}\left(\lambda_{1}-\lambda_{2}\right)\right|\right\}
$$


and $\operatorname{cum}\left\{I_{a_{1} b_{1}}^{(T)}\left(\lambda_{1}\right), \ldots, I_{a_{k} b_{k}}^{(T)}\left(\lambda_{k}\right)\right\}$

$$
=T^{-k} \Sigma \Delta^{(T)}\left(\mu_{1}+\nu_{1}\right) \ldots \Delta^{(T)}\left(\mu_{k}+\nu_{k}\right) f_{c_{1} d_{1}}\left(\mu_{1}\right) \ldots f_{c_{k} d_{k}}\left(\mu_{k}\right)+O\left(T^{-1}\right) .
$$

Here the summation in $(3 \cdot 5)$ extends over all partitions

$$
\left\{\left(c_{1}, \mu_{1}\right),\left(d_{1}, \nu_{1}\right)\right\}, \ldots,\left\{\left(c_{k}, \mu_{k}\right),\left(d_{k}, \nu_{k}\right)\right\}
$$

into pairs, of the quantities

$$
\left(a_{1}, \lambda_{1}\right),\left(b_{1},-\lambda_{1}\right), \ldots,\left(a_{k}, \lambda_{k}\right),\left(b_{k},-\lambda_{k}\right)
$$

excluding the cases with $\mu_{j}=-\nu_{j}=\lambda_{m}$ for some $j, m$. The error term, $O\left(T^{-1}\right)$, in $(3 \cdot 4)$ is uniform in $\lambda_{1}, \ldots, \lambda_{k}$.

The proofs are given in $\S 8$.

From $(3 \cdot 4)$ we can derive the following corollary.

CoRollary. Under the conditions of the theorem, if one of $\lambda_{j}+\lambda_{m} \equiv 0(\bmod 2 \pi)$ or $\lambda_{j}-\lambda_{m} \equiv 0(\bmod 2 \pi)$ is not true for each $j, m=1, \ldots, r$, then

$$
\operatorname{cum}\left\{I_{a_{1} b_{1}}^{(T)}\left(\lambda_{1}\right), \ldots, I_{a_{k} b_{k}}^{(T)}\left(\lambda_{k}\right)\right\} \rightarrow 0 \quad \text { as } \quad T \rightarrow \infty \quad(k=3,4, \ldots) .
$$

Let us now determine the limiting distribution of $\mathbf{I}_{X X}^{T\rangle_{X}}(\lambda)$ on the basis of the limiting values of its cumulants. We have,

Theorem 3.2. Let $\mathbf{X}(t)$ satisfy Assumption I and have mean 0 .

If $0 \leqslant \lambda_{1}<\lambda_{2}<\ldots<\lambda_{k} \leqslant \pi$, then $\mathbf{I}_{X X}^{(T)}\left(\lambda_{1}\right), \ldots, \mathbf{I}_{X X}^{(T)}\left(\lambda_{k}\right)$ are asymptotically independent. If $\lambda \neq 0(\bmod \pi)$, then $\mathbf{I}_{X X}^{(T)}(\lambda)$ tends, in distribution, to $W_{r}^{C}\left\{1, \mathbf{f}_{X X}(\lambda)\right\}$. If $\lambda \equiv 0(\bmod \pi)$, then it tends in distribution to $W_{r}\left\{1, \mathbf{f}_{X X}(\lambda)\right\}$.

The different asymptotic distributions in the cases $\lambda \neq 0(\bmod \pi)$ and $\lambda \equiv 0(\bmod \pi)$ reflect the fact that $\mathbf{f}_{X X}(\lambda)$ and $\mathbf{I}_{X X}^{(T)}(\lambda)$ are real-valued in the latter case.

The asymptotic behaviour of the periodogram, $I_{a a}^{(T)}(\lambda)$, of $X_{a}(t)$ has been considered by Bartlett (1966, p. 304), Grenander \& Rosenblatt (1957), Hannan (1960, p. 52) and Kawata (1959). Walker (1965) determines the asymptotic distribution of $I_{a a}^{(T)}(\lambda)$ for $X_{a}(t)$ a linear process. Rao (1967) considers asymptotic properties of the cross-periodogram $I_{a b}^{(T)}(\lambda), a \neq b$ of $X_{a}(t)$ and $X_{b}(t)$; see also Slutsky (1934) and Olshen (1967).

\section{The SPECTRAL Measure}

If the series $\mathbf{X}(t)(t=0, \pm 1, \ldots)$ has spectral density matrix $\mathbf{f}_{X X}(\lambda)$, then $\mathbf{F}_{X X}(\lambda)$, the matrix of spectral measures, is given by

$$
\mathbf{F}_{X X}(\lambda)=\int_{0}^{\lambda} \mathbf{f}_{X X}(\alpha) d \alpha \quad(0 \leqslant \lambda \leqslant \pi)
$$

Outside this range $\mathbf{F}_{X X}(\lambda)$ is taken to have period $2 \pi$ and satisfy $\mathbf{F}_{X X}(-\lambda)=\overline{\mathbf{F}}_{X X}^{\prime}(\lambda)$. In view of $(3 \cdot 1)$ one can consider estimating $\mathbf{F}_{X X}(\lambda)$ by

$$
\mathbf{F}_{X X}^{(T)}(\lambda)=\int_{0}^{\lambda} \mathbf{I}_{X X}^{(T)}(\alpha) d \alpha \quad(0 \leqslant \lambda \leqslant \pi) .
$$

Because of its elementary dependence on $\mathbf{I}_{X X}^{\left(\frac{T}{X}\right)}(\lambda)$, we can determine the asymptotic moments of $\mathbf{F}_{X X}^{(T)}(\lambda)$ directly from Theorem $3 \cdot 1$. We have 
TheOrem 4.1. Let $\mathbf{X}(t)$ satisfy Assumption I and have mean 0. Then

$$
\begin{gathered}
E\left\{F_{a_{1} b_{1}}^{(T)}\left(\lambda_{1}\right)\right\}=F_{a_{1} b_{1}}\left(\lambda_{1}\right)+O\left(T^{-1}\right), \\
\operatorname{cov}\left\{F_{a_{1} b_{1}}^{(T)}\left(\lambda_{1}\right), F_{a_{2} b_{2}}^{(T)}\left(\lambda_{2}\right)\right\}=2 \pi T^{-1}\left\{\int_{0}^{\min \left(\lambda_{1}, \lambda_{2}\right)} f_{a_{1} a_{2}}(\alpha) f_{b_{1} b_{2}}(-\alpha) d \alpha\right. \\
\left.\quad+\int_{0}^{\lambda_{1}} \int_{0}^{\lambda_{2}} f_{a_{1} b_{1} a_{2} b_{2}}(\alpha,-\alpha,-\beta) d \alpha d \beta\right\}+O\left(T^{-1} \log T\right) \\
\text { and } \quad \operatorname{cum}\left\{F_{a_{1} b_{1}}^{(T)}\left(\lambda_{1}\right), \ldots, F_{a_{k} b_{k}}^{(T)}\left(\lambda_{k}\right)\right\}=O\left(T^{-k+1}\right) \quad(k=1,2, \ldots) .
\end{gathered}
$$

We see that $\mathbf{F}_{X X}^{(T)}(\lambda)$ is an asymptotically unbiased and consistent estimate of $\mathbf{F}_{X X}(\lambda)$. In fact one has

COROLLARY. Under the conditions of the theorem

$$
\operatorname{prob}\left\{\lim _{T \rightarrow \infty} \mathbf{F}_{X X}^{\left(\frac{T}{X}\right)}(\lambda)=\mathbf{F}_{X X}(\lambda)\right\}=1 \quad(0 \leqslant \lambda \leqslant \pi)
$$

and, in fact,

$$
\operatorname{prob}\left\{\lim _{T \rightarrow \infty} \sup _{0 \leqslant \lambda \leqslant \pi}\left|\mathbf{F}_{X X}^{(T)}(\lambda)-\mathbf{F}_{X X}(\lambda)\right|=0\right\}=1 .
$$

We see that $\mathbf{F}_{X X}^{(T)}(\lambda)$ is a strongly consistent estimate of $\mathbf{F}_{X X}(\lambda)$ with the convergence uniform in $\lambda$.

Let us now turn to the consideration of the asymptotic distribution of $\mathbf{F}_{X X}^{(T)}(\lambda)$. We may use Theorem $4 \cdot 1$ to evaluate the limits of the cumulants of $T^{\frac{1}{2}}\left\{\mathbf{F}_{X X}^{(T)}(\lambda)-\mathbf{F}_{X X}(\lambda)\right\}$. The limits of cumulants of order greater than two are seen to vanish and we may conclude

Theorem 4.2. Let $\mathbf{X}(t)(t=0, \pm 1, \ldots)$ satisfy Assumption $I$ and have mean 0 . Then

$$
T^{\frac{1}{2}}\left\{\mathbf{F}_{X X}^{(T)}\left(\lambda_{1}\right)-\mathbf{F}_{X X}\left(\lambda_{1}\right)\right\}, \ldots, T^{\frac{1}{2}}\left\{\mathbf{F}_{X X}^{(T)}\left(\lambda_{k}\right)-\mathbf{F}_{X X}\left(\lambda_{k}\right)\right\}
$$

are asymptotically jointly multivariate normal with covariance structure given by

$$
\begin{aligned}
& \lim _{T \rightarrow \infty} \operatorname{cov}\left[T^{\frac{1}{2}}\left\{F_{a_{1} b_{1}}^{(T)}\left(\mu_{1}\right)-F_{a_{1} b_{1}}\left(\mu_{1}\right)\right\}, T^{\frac{1}{2}}\left\{F_{a_{2} b_{2}}^{(T)}\left(\mu_{2}\right)-F_{a_{2} b_{2}}\left(\mu_{2}\right)\right\}\right] \\
& =2 \pi \int_{0}^{\min \left(\mu_{1}, \mu_{2}\right)} f_{a_{1} a_{2}}(\alpha) f_{b_{1} b_{2}}(-\alpha) d \alpha+2 \pi \int_{0}^{\mu_{1}} \int_{0}^{\mu_{2}} f_{a_{1} b_{1} a_{2} b_{2}}(\alpha,-\alpha, \beta) d \alpha d \beta \\
& \left(\mu_{1}, \mu_{2}=\lambda_{1}, \ldots, \lambda_{k} ; a_{j}, b_{j}=1,2, \ldots, r ; j=1,2, \ldots, k ; k=1,2, \ldots\right) .
\end{aligned}
$$

This theorem indicates the asymptotic normality of finite collections of the $\mathbf{F}_{X X}^{(T)}(\lambda)$. We turn to stronger results concerning the convergence of the stochastic process

$$
\left[T^{\frac{1}{2}}\left\{\mathbf{F}_{X X}^{(T)}(\lambda)-\mathbf{F}_{X X}(\lambda)\right\} \quad(0 \leqslant \lambda \leqslant \pi)\right]
$$

to a certain Gaussian process. We first set down some terminology.

For $0<\alpha \leqslant 1, \operatorname{Lip}_{\alpha}^{r \times r}(0, \pi)$ will denote the Banach space of $r \times r$ matrix-valued functions $\mathbf{Y}(\lambda)(0<\lambda \leqslant \pi), \mathbf{Y}(0)=0$, with norm

$$
\|\mathbf{Y}(\lambda)\|=\sup _{0 \leqslant \lambda<\pi}|\mathbf{Y}(\lambda)|+\sup _{0 \leqslant \lambda, \lambda+\epsilon \leqslant \pi}|\epsilon|^{-\alpha}|\mathbf{Y}(\lambda+\epsilon)-\mathbf{Y}(\lambda)| .
$$

In the case $r=1$, this space is discussed by Lamperti (1962). 
A sequence $\left\{\mathbf{Y}^{(T)}(\lambda)(0 \leqslant \lambda \leqslant \pi)\right\}(T=1,2, \ldots)$ of stochastic processes, with values in $\operatorname{Lip}_{\alpha}^{r \times r}(0, \pi)$, is said to converge weakly in the topology of $\operatorname{Lip}_{\alpha}^{r \times r}$ to a process $\mathbf{Y}(\lambda)$, with values in $\operatorname{Lip}_{\alpha}^{r \times r}(0, \pi)$, if

$$
\lim _{T \rightarrow \infty} E\left[\mathscr{F}\left\{\mathbf{Y}^{(T)}\right\}\right]=E\{\mathscr{F}(\mathbf{Y})\}
$$

for any bounded real-valued function $\mathscr{F}($.$) , continuous on \operatorname{Lip}_{\alpha}^{r \times r}(0, \pi)$.

We may now state

Theorem 4 $\cdot 3$. Let $\mathbf{X}(t)(t=0, \pm 1, \ldots)$ satisfy Assumption I and have mean 0. Then, for any $\alpha$ with $0<\alpha<\frac{1}{2}$, the sequence of processes

$$
\left[T^{\frac{1}{2}}\left\{\mathbf{F}_{X X}^{(T)}(\lambda)-\mathbf{F}_{X X}(\lambda)\right\} \quad(0 \leqslant \lambda \leqslant \pi)\right]
$$

converges weakly in the topology of $\operatorname{Lip}_{\alpha}^{r \times r}$ to an $r \times r$ matrix-valued Gaussian process

with mean 0 and

$$
\{\mathbf{Y}(\lambda) \quad(0 \leqslant \lambda \leqslant \pi)\}
$$

$$
\begin{aligned}
\operatorname{cov}\left\{Y_{a_{1} b_{1}}\left(\lambda_{1}\right),\right. & \left.Y_{a_{2} b_{2}}\left(\lambda_{2}\right)\right\}=2 \pi \int_{0}^{\min \left(\lambda_{1}, \lambda_{2}\right)} f_{a_{1} a_{2}}(\alpha) f_{b_{1} b_{2}}(-\alpha) d \alpha \\
& +2 \pi \int_{0}^{\lambda_{1}} \int_{0}^{\lambda_{2}} f_{a_{1} b_{1} a_{2} b_{2}}(\alpha,-\alpha,-\beta) d \alpha d \beta \quad\left(a_{j}, b_{j}=1,2, \ldots, r ; j=1,2\right) .
\end{aligned}
$$

If $\mathbf{X}(t)$ satisfies the condition of the theorem, we are now able to assert the convergence in distribution of functionals such as

$$
\begin{gathered}
T^{\frac{1}{2}} \sup _{0 \leqslant \lambda \leqslant \pi}\left|\mathbf{F}_{X X}^{(T)}(\lambda)-\mathbf{F}_{X X}(\lambda)\right| \\
T \int_{0}^{\pi} \int_{0}^{\pi}|\alpha-\beta|^{-\frac{1}{2}}\left|\mathbf{F}_{X X}^{\left(\frac{T}{X}\right)}(\alpha)-\mathbf{F}_{X X}^{(T)}(\beta)-\mathbf{F}_{X X}(\alpha)+\mathbf{F}_{X X}(\beta)\right|^{2} d \alpha d \beta
\end{gathered}
$$

to corresponding functionals based on the Gaussian process $\mathbf{Y}(\lambda)$ of the theorem.

Because

$$
\mathbf{I}_{X X}^{(T)}(\lambda)=\frac{d}{d \lambda}\left\{\mathbf{F}_{X X}^{(T)}(\lambda)\right\} \quad(0 \leqslant \lambda \leqslant \pi)
$$

we may expect $\mathbf{I}_{X X}^{(T)}(\lambda)$ to exhibit some of the properties of the (generalized) derivative of the process $\mathbf{Y}(\lambda)$.

If $r=1$ and $X(t)$ is a linear process, then Grenander \& Rosenblatt (1957) demonstrated the weak convergence of $T^{\frac{1}{2}}\left|F^{(T)}(\lambda)-F(\lambda)\right|$ to a Gaussian process in the coarser topology of uniform convergence. Ibragimov (1963) and Malevich $(1964,1965)$ have considered the weak convergence of $T^{\frac{1}{2}}\left|F^{(T)}(\lambda)-F(\lambda)\right|$ in the case that $X(t)$ is Gaussian with square integrable spectral density.

\section{The autocovariance function}

Let $\mathbf{X}(t)(t=0, \pm 1, \ldots)$ denote an $r$ vector-valued stationary series with autocovariance function

$$
\mathbf{c}_{X X}(u)=E\left([\mathbf{X}(t+u)-E\{\mathbf{X}(t+u)\}][\mathbf{X}(t)-E\{\mathbf{X}(t)\}]^{\prime}\right) \quad(u=0, \pm 1, \ldots) .
$$

If $E\{\mathbf{X}(t)\}=0$, and the values $\mathbf{X}(t)(t=0,1, \ldots, T-1)$ are available, then we can consider estimating $\mathbf{c}_{X X}(u)$ by

$$
\mathbf{m}_{X X}^{(T)}(u)=T^{-1} \sum_{0 \leqslant t, t+u \leqslant T-1} \mathbf{X}(t+u) \mathbf{X}^{\prime}(t) \quad(u=0, \pm 1, \ldots) .
$$


We have seen that

$$
\mathbf{m}_{X X}^{(T)}(u)=\int_{-\pi}^{\pi} \mathbf{I}_{X X}^{(T)}(\alpha) \exp (i u \alpha) d \alpha
$$

and so we may determine the statistical properties of $\mathbf{m}_{X X}^{\left(T^{\prime}\right)}(u)$ from those of $\mathbf{I}_{X X}^{(T)}(\alpha)$. Theorem $3 \cdot 1$ gives directly

TheOREm 5·1. Let $\mathbf{X}(t)(t=0, \pm 1, \ldots)$ satisfy Assumption $I$ and have mean 0. Then

$$
E\left\{m_{a_{1} b_{1}}^{(T)}\left(u_{1}\right)\right\}=m_{a_{1} b_{1}}\left(u_{1}\right)+O\left(T^{-1}\right),
$$

$$
\begin{aligned}
& \operatorname{cov}\left\{m_{a_{1} b_{1}}^{(T)}\left(u_{1}\right), m_{a_{2} b_{2}}^{(T)}\left(u_{2}\right)\right\} \\
&= T^{-1}\left[\int_{0}^{2 \pi} \exp \left\{i \alpha\left(u_{1}-u_{2}\right)\right\} f_{a_{1} a_{2}}(\alpha) f_{b_{1} b_{2}}(-\alpha) d \alpha\right. \\
&+\int_{0}^{2 \pi} \exp \left\{i \alpha\left(u_{1}+u_{2}\right)\right\} f_{a_{1} b_{2}}(\alpha) f_{b_{1} a_{2}}(-\alpha) d \alpha \\
&\left.+2 \pi \int_{0}^{2 \pi} \int_{0}^{2 \pi} \exp \left\{i\left(\alpha_{1} u_{1}+\alpha_{2} u_{2}\right)\right\} f_{a_{1} b_{1} a_{2} b_{2}}\left(\alpha_{1},-\alpha_{1}, \alpha_{2}\right) d \alpha_{1} d \alpha_{2}\right]+O\left(T^{-2} \log T\right)
\end{aligned}
$$

and

$$
\operatorname{cum}\left\{m_{a_{1} b_{1}}^{(T)}\left(u_{1}\right), \ldots, m_{a_{k} b_{k}}^{(T)}\left(u_{k}\right)\right\}=O\left(T^{-k+1}\right)
$$

for $u_{j}=0, \pm 1, \ldots ; a_{j}, b_{j}=1,2, \ldots, r ; j=1, \ldots, k$ and $k=1,2, \ldots$ The error terms are uniform in each case.

CoRollary. Under the conditions of the theorem

$$
\operatorname{prob}\left\{\lim _{T \rightarrow \infty} \mathbf{m}_{X X}^{\left(T^{T}\right)}(u)=\mathbf{m}_{X X}(u)\right\}=1 \quad(u=0, \pm 1, \ldots) .
$$

Also

$$
\operatorname{prob}\left[\lim _{T \rightarrow \infty} \sup _{u \neq 0}\left|u^{-1}\left\{\mathbf{m}_{X X}^{(T)}(u)-\mathbf{m}_{X X}(u)\right\}\right|=0\right]=1 .
$$

Let us now turn to an investigation of the asymptotic distribution of finite collections of the $\mathbf{m}_{X X}^{\left(T^{\prime}\right)}(u)$.

TheOREM 5•2. Let $X(t)(t=0, \pm 1, \ldots)$ satisfy Assumption $I$ and have mean 0 . Then

$$
T^{\frac{1}{2}}\left\{\mathbf{m}_{X X}^{(T)}\left(u_{1}\right)-\mathbf{m}_{X X}\left(u_{1}\right)\right\}, \ldots, T^{\frac{1}{2}}\left\{\mathbf{m}_{X X}^{(T)}\left(u_{k}\right)-\mathbf{m}_{X X}\left(u_{k}\right)\right\}
$$

are asymptotically jointly multivariate normal with covariance structure given by

$$
\begin{aligned}
\lim _{T \rightarrow \infty} \operatorname{cov}[ & \left.T^{\frac{1}{2}}\left\{m_{a_{1} b_{1}}^{(T)}\left(u_{1}\right)-m_{a_{1} b_{1}}\left(u_{1}\right)\right\}, T^{\frac{1}{2}}\left\{m_{a_{2} b_{2}}^{(T)}\left(u_{2}\right)-m_{a_{2} b_{2}}\left(u_{2}\right)\right\}\right] \\
= & \int_{0}^{2 \pi} \exp \left\{i \alpha\left(u_{1}-u_{2}\right)\right\} f_{a_{1} a_{2}}(\alpha) f_{b_{1} b_{2}}(-\alpha) d \alpha+\int_{0}^{2 \pi} \exp \left\{i \alpha\left(u_{1}+u_{2}\right)\right\} f_{a_{1} b_{2}}(\alpha) f_{b_{1} a_{2}}(-\alpha) d \alpha \\
& +2 \pi \int_{0}^{2 \pi} \int_{0}^{2 \pi} \exp \left\{i\left(\alpha_{1} u_{1}+\alpha_{2} u_{2}\right)\right\} f_{a_{1} b_{1} a_{2} b_{2}}\left(\alpha_{1},-\alpha_{1}, \alpha_{2}\right) d \alpha_{1} d \alpha_{2} .
\end{aligned}
$$

Let us turn to the derivation of a theorem concerning the asymptotic behaviour of the function $\left\{\mathbf{m}_{X X}^{(T)}(u)(u=0, \pm 1, \ldots)\right\}$. We first introduce $\mathscr{A}_{\alpha}^{r \times r}$. This is the image of $\operatorname{Lip}_{\alpha}^{r \times r}(0, \pi)$ by the Fourier-Stieltjes transform, that is the space of $r \times r$ matrix-valued sequences $\{\mathbf{y}(u)(u=0, \pm 1, \ldots)\}$ of the form

$$
\mathbf{y}(u)=\int_{0}^{\pi} \exp (i u \alpha) d \mathbf{Y}(\alpha)
$$

where $\mathbf{Y}(\alpha) \in \operatorname{Lip}_{\alpha}^{r \times r}(0, \pi)$ for $0<\alpha \leqslant 1$. As norm of this $\mathbf{y}(u)$ we take $\|\mathbf{Y}(\lambda)\|$. 
Elementary calculations indicate that $\mathscr{A}_{\alpha}^{r \times r}$ is a subspace of the Banach space of $r \times r$ matrix-valued sequences, $\mathbf{y}(u)(u=0, \pm 1, \ldots)$, with norm

We have

$$
\|\mathbf{y}(u)\|_{\alpha}=\sup _{u}|\mathbf{y}(u)|+\sup _{u \neq 0}\left|u^{-1+\alpha} \mathbf{y}(u)\right| \text {. }
$$

Theorem 5•3. Let $\mathbf{X}(t)$ satisfy Assumption $I$ and have mean 0 . Then, for any $\alpha$ with $0<\alpha<\frac{1}{2}$, the sequence of processes $\left\{T^{\frac{1}{2}}\left\{\mathbf{m}_{X X}^{(T)}(u)-\mathbf{m}_{X X}(u)\right\}(u=0, \pm 1, \ldots)\right\}$ converges weakly in the topology of $\mathscr{A}_{\alpha}^{r \times r}$ to a zero mean Gaussian process $\{\mathbf{y}(u)(u=0, \pm 1, \ldots)\}$ with

$$
\begin{aligned}
\operatorname{cov} & \left\{y_{a_{1} b_{1}}\left(u_{1}\right), y_{a_{2} b_{2}}\left(u_{2}\right)\right\} \\
= & \int_{0}^{2 \pi} \exp \left\{i \alpha\left(u_{1}-u_{2}\right)\right\} f_{a_{3} a_{2}}(\alpha) f_{b_{1} b_{2}}(-\alpha) d \alpha+\int_{0}^{2 \pi} \exp \left\{i \alpha\left(u_{1}+u_{2}\right)\right\} f_{a_{1} b_{2}}(\alpha) f_{b_{1} a_{2}}(-\alpha) d \alpha \\
& \quad+2 \pi \int_{0}^{2 \pi} \int_{0}^{2 \pi} \exp \left\{i\left(\alpha_{1} u_{1}+\alpha_{2} u_{2}\right)\right\} f_{a_{1} b_{1} a_{2} b_{2}}\left(\alpha_{1},-\alpha_{1}, \alpha_{2}\right) d \alpha_{1} d \alpha_{2} .
\end{aligned}
$$

Turning to related work and the case of a real-valued series $X_{a}(t)$, we note that Slutsky (1934) considered asymptotic properties of $m_{a a}^{(T)}(u)$ in the Gaussian case. Parzen (1961) gave conditions for the convergence of $m_{a a}^{(T)}(u)$ with probability 1 . Bartlett (1946; 1966, p. 285) and Parzen (1957b) developed formulae for the asymptotic variance of $m_{a a}^{(T)}(u)$. Walker (1954), Lomnicki \& Zaremba (1957, 1959), Parzen (1957 a) and Anderson \& Walker (1964) considered the asymptotic normality of $m_{a a}^{(T)}(u)$ in the case where $X_{a}(t)$ was a linear process. Rosenblatt (1962) considered asymptotic normality in the case where $X_{a}(t)$ is Gaussian.

Bartlett (1966, p. 286) noted that, if instead of the autocovariance function $m_{a a}^{(T)}(u)$, one considered the autocorrelation function $m_{a a}^{(T)}(u) / m_{a a}^{(T)}(0)$, and $X_{a}(t)$ was a linear process, then only second-order spectra appear in the asymptotic variance formula. Elementary calculations based on $(5 \cdot 9)$ indicate that this result does not continue to hold in the case that $X_{a}(t)$ is not a linear process.

\section{The SPECTRAL DENSITY}

We turn to the investigation of estimates of $\mathbf{f}_{X X}(\lambda)$ the matrix of second-order spectral densities. Let $H(\alpha)(-\pi<\alpha \leqslant \pi)$ be a weight function satisfying Assumption II. Let $B_{T}$ be a scale factor depending on $T$. Suppose $\mathbf{X}(t)(t=0, \pm 1, \ldots)$ has mean $\mathbf{0}$. Let

$$
h^{(T)}(u)=\int_{-\pi}^{\pi} H^{(T)}(\alpha) \exp (-i u \alpha) d \alpha
$$

where $H^{(T)}(\alpha)$ is given by $(2 \cdot 11)$. As an estimate of $\mathbf{f}_{X X}^{(T)}(\alpha)$, we propose

$$
\begin{aligned}
\mathbf{f}_{X X}^{(T)}(\lambda) & =(2 \pi)^{-1} \sum_{u=-T+1}^{T-1} h^{\left(T^{\prime}\right)}(u) \mathbf{m}_{X X}^{(T)}(u) \exp (-i u \lambda) \\
& =\int_{-\pi}^{\pi} H^{(T)}(\alpha) \mathbf{I}_{X X}^{(T)}(\lambda-\alpha) d \alpha
\end{aligned}
$$

that is a weighted average of the periodogram. We may prove

Theorem 6 $\cdot 1$. Let $\mathbf{X}(t)(t=0, \pm 1, \ldots)$ satisfy Assumption $I$ and have mean 0 . Let $\mathbf{f}_{X X}^{(T)}(\lambda)$ be constructed in the manner of $(6 \cdot 2)$, where $H(\alpha)$ satisfies Assumption II. Then

$$
E\left\{f_{a_{1} b_{1}}^{(T)}\left(\lambda_{1}\right)\right\}=\int_{-\pi}^{\pi} H(\alpha) f_{a_{1} b_{1}}\left(\lambda-B_{T} \alpha\right) d \alpha+O\left(T^{-1}\right)
$$




$$
\begin{aligned}
& \operatorname{cov}\left\{f_{a_{1} b_{1}}^{(T)}\left(\lambda_{1}\right), f_{a_{2} b_{2}}^{(T)}\left(\lambda_{2}\right)\right\} \\
&= T^{-1}\left\{\int_{-\pi}^{\pi} H(\alpha) H\left(\lambda_{2}-\lambda_{1}-\alpha\right) f_{a_{1} a_{2}}\left(\lambda_{1}-\alpha\right) f_{b_{1} b_{2}}\left(\alpha-\lambda_{1}\right) d \alpha\right. \\
&\left.+\int_{-\pi}^{\pi} H(\alpha) H\left(\lambda_{2}+\lambda_{1}-\alpha\right) f_{a_{1} b_{2}}\left(\lambda_{1}-\alpha\right) f_{b_{1} a_{2}}\left(\alpha-\lambda_{1}\right) d \alpha\right\} \\
&+2 \pi T^{-1} \iint_{-\pi}^{\pi} H\left(\alpha_{1}\right) H\left(\alpha_{2}\right) f_{a_{1} b_{1} a_{2} b_{2}}\left(\lambda_{1}-\alpha_{1}, \alpha_{1}-\lambda_{1}, \alpha_{2}-\lambda_{2}\right) d \alpha_{1} d \alpha_{2} \\
&+O\left(T^{-2} \log T\right) \quad\left(B_{T}=1\right)
\end{aligned}
$$

$$
\begin{aligned}
\operatorname{cov}\left\{f_{a_{1} b_{1}}^{(T)}\left(\lambda_{1}\right), f_{a_{2} b_{2}}^{(T)}\left(\lambda_{2}\right)\right\}= & B_{T}^{-1} T^{-1}\left\{\int_{-\pi}^{\pi} H(\alpha)^{2} d \alpha\right\}\left[\eta\left(\lambda_{1}-\lambda_{2}\right) f_{a_{1} a_{2}}\left(\lambda_{1}\right) f_{b_{1} b_{2}}\left(-\lambda_{1}\right)\right. \\
& \left.+\eta\left(\lambda_{1}+\lambda_{2}\right) f_{a_{1} b_{2}}\left(\lambda_{1}\right) f_{b_{1} a_{2}}\left(-\lambda_{1}\right)\right] \\
& +O\left(B_{T}^{-2} T^{-2}\right) \quad\left(B_{T} \rightarrow 0, B_{T} T \rightarrow \infty \text { as } T \rightarrow \infty\right) .
\end{aligned}
$$

Also $\operatorname{cum}\left\{f_{a_{1} b_{1}}^{(T)}\left(\lambda_{1}\right), \ldots, f_{a_{k} b_{k}}^{(T)}\left(\lambda_{k}\right)\right\}= \begin{cases}O\left(T^{-k+1}\right) & \left(B_{T}=1\right), \\ O\left(B_{T}^{-k+1} T^{-k+1}\right) & \left(B_{T} \rightarrow 0, B_{T} T \rightarrow \infty \text { as } T \rightarrow \infty\right) .\end{cases}$

Turning to the asymptotic distribution of $\mathbf{f}_{X X}^{(T)}(\lambda)$, we have

Theorem 6 2. Let $\mathbf{X}(t)(t=0, \pm 1, \ldots)$ satisfy Assumption $I$ and have mean 0 . Let

$$
\mathbf{f}_{X X}^{(T)}\left(\lambda_{1}\right), \ldots, \mathbf{f}_{X X}^{(T)}\left(\lambda_{k}\right)
$$

be constructed in the manner of (6.2), where $H(\alpha)$ satisfies Assumption II. If $B_{T} T \rightarrow \infty$ as $T \rightarrow \infty$, then

$$
\left(B_{T} T\right)^{\frac{1}{2}}\left[\mathbf{f}_{X X}^{(T)}\left(\lambda_{1}\right)-E\left\{\mathbf{f}_{X X}^{(T)}\left(\lambda_{1}\right)\right\}\right], \ldots,\left(B_{T} T\right)^{\frac{1}{2}}\left[\mathbf{f}_{X X}^{(T)}\left(\lambda_{k}\right)-E\left\{\mathbf{f}_{X X}^{(T)}\left(\lambda_{k}\right)\right\}\right] \quad(k=1,2, \ldots)
$$

is asymptotically normal with mean 0 and covariance structure indicated by $(6 \cdot 4)$.

On occasion an alternative form of asymptotic distribution may proverelevant. Suppose we estimate $\mathbf{f}_{X X}(\lambda)$ by a simple average of periodograms. For example, with $s(T), m$ integers and $2 \pi s(T) / T$ near $\lambda$, consider

$$
(2 m+1)^{-1} \sum_{s=-m}^{m} \mathbf{I}_{X X}^{(T)}[2 \pi\{s(T)+s\} / T] \quad(\lambda \neq 0, \bmod \pi)
$$

and

$$
(2 m+2)^{-1}\left\{\mathbf{I}_{X X}^{(T)}(\lambda)+\sum_{s=-m}^{m} \mathbf{I}_{X X}^{(T)}(\lambda+2 \pi s / T)\right\} \quad(\lambda \equiv 0, \bmod \pi)
$$

Then one has

Theorem 6 3. Let $\mathbf{X}(t)(t=0, \pm 1, \ldots)$ satisfy Assumption $I$ and have mean 0. Let $m$ be fixed and $2 \pi s(T) / T \rightarrow \lambda$ as $T \rightarrow \infty$. If $\lambda \neq 0(\bmod \pi),(6 \cdot 5)$ tends in distribution to

$$
(2 m+1)^{-1} W_{r}^{C}\left\{2 m+1, \mathbf{f}_{X X}(\lambda)\right\} \text {. }
$$

If $\lambda \equiv 0(\bmod \pi),(6 \cdot 6)$ tends in distribution to $(2 m+1)^{-1} W_{r}\left\{2 m+1, f_{X X}(\lambda)\right\}$.

In the notation of $(6 \cdot 2)$, the estimates $(6 \cdot 5),(6 \cdot 6)$ correspond to a $B_{T}$ of order $T^{-1}$.

One may prove a theorem concerning the weak convergence of the process

$$
\left\{\mathbf{f}_{X X}^{(T)}(\lambda) \quad(-\infty<\lambda<\infty)\right\}
$$

in the case $B_{T}=1$. The theorem follows directly from the weak convergence of

$$
\left\{\mathbf{F}_{X X}^{(T)}(\lambda) \quad(0 \leqslant \lambda \leqslant \pi)\right\}
$$


and the representation

$$
f_{a b}^{(T)}(\lambda)=\int_{0}^{\pi} H(\alpha) d\left\{F_{a b}^{(T)}(\lambda-\alpha)+F_{b a}^{(T)}(\lambda-\alpha)\right\}
$$

The theorem involves the weak convergence of $T^{\frac{1}{2}}\left[\mathbf{f}_{X X}^{(T)}(\lambda)-E\left\{\mathbf{f}_{X X}^{(T)}(\lambda)\right\}\right]$ to a zero mean Gaussian process with covariance structure indicated by $(6 \cdot 4)$ and is clear in view of our previous results.

The asymptotic mean and variance of power spectral estimates were investigated by Grenander \& Rosenblatt (1957), Parzen (1957a,b) and Blackman \& Tukey (1958, p. 16). Asymptotic normality has been demonstrated, under various conditions, by Rosenblatt (1959) and Brillinger (1965, 1968). Bartlett (1950) made use of the $\chi^{2}$ distribution for smoothed periodogram estimates. The approximation of the distribution of $f_{X X}^{\left(T^{\prime}\right)}(\lambda)$ by a complex Wishart was suggested by Goodman (1963). Wahba (1968) proves that expression $(6 \cdot 5)$ has the form $(2 m+1)^{-1} W_{r}^{C}\left\{2 m+1, \mathbf{f}_{X X}(\lambda)\right\}+O_{p}\left(T^{-1}\right)+O_{p}\left(m^{-1}\right)$. This does not yield our Theorem $6 \cdot 3$, however.

\section{Departures from assumptions}

The most common departure from the assumptions of this paper will be for the series to have non-zero mean.

Let $\mathbf{Y}(t)$ satisfy Assumption I. Let $\mathbf{X}(t)=\mathbf{Y}(t)-E\{\mathbf{Y}(t)\}$, then $\mathbf{X}(t)$ will have zero mean and the results of the paper will apply to it. Suppose $\mathbf{Y}(t)(t=0,1, \ldots, T-1)$ are available. Set

and

$$
\begin{gathered}
\overline{\mathbf{Y}}^{(T)}=T^{-1} \sum_{t=0}^{T-1} \mathbf{Y}(t) \quad(t=0,1, \ldots, T-1) \\
\mathbf{Y}^{(T)}(t)=\mathbf{Y}(t)-\overline{\mathbf{Y}}^{(T)}=\mathbf{X}(t)+\left[E\{\mathbf{Y}(t)\}-\overline{\mathbf{Y}}^{(T)}\right] \quad(t=0,1, \ldots, T-1) .
\end{gathered}
$$

Our procedure will be to replace the statistics, of the paper, based on $\mathbf{X}(t)(t=0,1, \ldots, T-1)$ by statistics based on $\mathbf{Y}^{\left(T^{\prime}\right)}(t)(t=0,1, \ldots, T-1)$.

In many cases the difference $\overline{\mathbf{Y}}^{(T)}-E\{\mathbf{Y}(t)\}$ is asymptotically negligible and the results of the paper continue to hold. See the discussion of Walker (1965), Parzen $(1957 a, b)$, Hannan (1967), for example.

There are immediate extensions of the theorems of this paper to apply to the case of a continuous time process $\mathbf{X}(t)(-\infty<t<\infty)$ satisfying

$$
\int_{t_{1}, \ldots, t_{k-1}} \ldots \int_{j}\left|t_{j} c_{a_{1}, \ldots, a_{k}}\left(t_{1}, \ldots, t_{k-1}\right)\right| d t_{1} \ldots d t_{k-1}<\infty \quad\left(a_{1}, \ldots, a_{k}=1,2, \ldots, r ; k=1,2, \ldots\right) .
$$

\section{Proofs}

In this section we present proofs of the various theorems of the paper.

Proof of Theorem 3.1. Let us determine

$$
\operatorname{cum}\left\{d_{a_{1}}^{(T)}\left(\lambda_{1}\right) d_{b_{1}}^{(T)}\left(-\lambda_{1}\right), \ldots, d_{a_{k}}^{(T)}\left(\lambda_{k}\right) d_{b_{k}}^{(T)}\left(-\lambda_{k}\right)\right\}
$$

as $(3 \cdot 1),(3 \cdot 2),(3 \cdot 3)$ follow directly from it. We use a result of Leonov $\&$ Shiryaev (1959) and argue as did Brillinger (1965), Brillinger \& Rosenblatt (1967) and Brillinger (1968). The cumulant in question is given by

$$
\sum_{\nu} \operatorname{cum}\left(C_{\nu_{1}}\right) \ldots \operatorname{cum}\left(C_{\nu_{p}}\right)
$$


where $\left[C_{\nu_{1}}, \ldots, C_{\nu_{p}}\right]$ is an indecomposable partition of the elements of the table

$$
\begin{array}{cc}
d_{a_{1}}^{(T)}\left(\lambda_{1}\right), & d_{b_{1}}^{(T)}\left(-\lambda_{1}\right) \\
\vdots & \vdots \\
d_{a_{k}}^{(T)}\left(\lambda_{k}\right), & d_{b_{k}}^{(T)}\left(-\lambda_{k}\right)
\end{array}
$$

and the summation in (8.1) extends over all such indecomposable partitions. We may now use Lemma $2 \cdot 1$ to evaluate cum $\left(C_{y_{j}}\right)$ and obtain $(3 \cdot 1),(3 \cdot 2),(3 \cdot 3)$ by retaining only the principal terms.

Proof of Corollary. This follows from the fact that $T^{-1}\left|\Delta^{(T)}(\lambda)\right| \rightarrow 0$ unless $\lambda \equiv 0(\bmod 2 \pi)$.

Before turning to a proof of Theorem $3 \cdot 2$, we first note that the characteristic function of a $W_{r}(\nu, \Sigma)$ variate is given by

$$
\operatorname{det}(\mathbf{I}-2 i \boldsymbol{\Sigma} \boldsymbol{\Theta})^{-\frac{1}{2} \nu}
$$

(Anderson, 1958), while that of a $W_{r}^{C}(\nu, \boldsymbol{\Sigma})$ variate is given (Goodman, 1963) by

$$
\operatorname{det}(\mathbf{I}-i \boldsymbol{\Sigma} \boldsymbol{\Theta})^{-\nu} \text {. }
$$

These are both analytic in a neighbourhood of the origin so the variates are determined by their moments.

We can now turn to

Proof of Theorem $3 \cdot 2$. The stated asymptotic independence follows from the Corollary of Theorem $3 \cdot 1$.

Suppose $\lambda \neq 0(\bmod \pi)$, then from $(3 \cdot 4)$, it follows that

$$
\lim _{T \rightarrow \infty} \operatorname{cum}\left\{I_{a_{1} b_{1}}^{(T)}(\lambda), \ldots, I_{a_{k} b_{k}}^{(T)}(\lambda)\right\}=\Sigma f_{c_{1} d_{1}}(\lambda) \ldots f_{c_{k} d_{k}}(\lambda)
$$

where the summation in (8.5) extends over permutations $\left(c_{1}, \ldots, c_{k}\right)$ of $\left(a_{1}, \ldots, a_{k}\right)$, permutations $\left(d_{1}, \ldots, d_{k}\right)$ of $\left(b_{1}, \ldots, b_{k}\right)$, no $d_{j}=b_{m}$ if $a_{j}=a_{m}$. The rules of Leonov \& Shiryaev indicate that (8.5) is cum $\left(W_{a_{1} b_{\mathbf{1}}}, \ldots, W_{a_{k} b_{k}}\right)$, where $\mathbf{W}$ is $W_{r}^{C}\left\{1, \mathbf{f}_{X X}(\lambda)\right\}$. Because the complex Wishart is determined by its moments, the proof is completed in the case $\lambda \neq 0(\bmod \pi)$. The case $\lambda \equiv 0(\bmod \pi)$ follows in a similar manner.

Before proceeding to the proof of Theorem $4 \cdot 1$, we note two properties of the function $\Delta^{(T)}(\alpha)$.

To begin, we have (Edwards, 1967, p. 80)

$$
\int_{0}^{\pi}\left|\Delta^{(T)}\left(\alpha_{2}-\alpha_{1}\right)\right| d \alpha_{2}=O(\log T)
$$

Also for $0 \leqslant \alpha_{1}, \lambda_{2} \leqslant \pi$

$$
\int_{0}^{\lambda_{2}} T^{-1}\left|\Delta^{(T)}\left(\alpha_{1}-\alpha_{2}\right)\right|^{2} d \alpha_{2}=\left\{\begin{array}{ll}
O\left(T^{-1}\right) & \left(\lambda_{2}<\alpha_{1}\right) \\
2 \pi+O\left(T^{-1}\right) & \left(\lambda_{2}>\alpha_{1}\right)
\end{array}\right\}
$$

This last expression follows from the fact (Edwards, 1967, p. 79) that

$$
\int_{0}^{2 \pi} T^{-1}\left|\Delta^{(T)}(\alpha)\right|^{2} d \alpha=2 \pi
$$

Proof of Theorem $4 \cdot 1$. We note that

$$
F_{a_{j} b_{j}}^{(T)}\left(\lambda_{j}\right)=\int_{0}^{\lambda_{j}} I_{a_{j} b_{j}}^{(T)}\left(\alpha_{j}\right) d \alpha_{j} \quad(j=1,2, \ldots, k),
$$

and so results may be made to follow from corresponding results concerning $I_{a_{j} b_{j}}^{(T)}\left(\alpha_{j}\right)$. 
Relation $(4 \cdot 3)$ is seen to follow directly from relations $(4 \cdot 2)$ and $(3 \cdot 1)$. Turning to $(4 \cdot 4)$, from $(3 \cdot 2)$ one has

$$
\begin{aligned}
& \operatorname{cov}\left\{F_{a_{1} b_{1}}^{(T)}\left(\lambda_{1}\right), F_{a_{2} b_{2}}^{(T)}\left(\lambda_{2}\right)\right\} \\
&= \int_{0}^{\lambda_{1}} f_{a_{1} a_{2}}\left(\alpha_{1}\right) f_{b_{1} b_{2}}\left(-\alpha_{1}\right)\left\{\int_{0}^{\lambda_{2}} T^{-2}\left|\Delta^{(T)}\left(\alpha_{1}-\alpha_{2}\right)\right|^{2} d \alpha_{2}\right\} d \alpha_{1} \\
&+\int_{0}^{\lambda_{1}} f_{a_{1} b_{2}}\left(\alpha_{1}\right) f_{b_{1} a_{2}}\left(-\alpha_{1}\right)\left\{\int_{0}^{\lambda_{2}} T^{-2}\left|\Delta^{(T)}\left(\alpha_{1}+\alpha_{2}\right)\right|^{2} d \alpha_{2}\right\} d \alpha_{1} \\
&+2 \pi T^{-1} \int_{0}^{\lambda_{1}} \int_{0}^{\lambda_{2}} f_{a_{1} b_{1} a_{2} b_{2}}\left(\alpha_{1},-\alpha_{1},-\alpha_{2}\right) d \alpha_{1} d \alpha_{2}+O\left(T^{-2} \log T\right)
\end{aligned}
$$

and the indicated result follows from the previous discussion.

Expression $(4 \cdot 5)$ follows from $(3 \cdot 4)$ directly.

Proof of Corollary. Equation $(4 \cdot 6)$ states that $\mathbf{F}_{X X}^{(T)}(\lambda)$ tends to $\mathbf{F}_{X X}(\lambda)$ with probability one. Now $(4 \cdot 3),(4 \cdot 4)$ and $(4 \cdot 5)$ indicate that

$$
E\left\{\left|F_{a_{1} b_{1}}^{(T)}\left(\lambda_{1}\right)-F_{a_{1} b_{1}}\left(\lambda_{1}\right)\right|^{4}\right\}=O\left(T^{-2}\right) \quad\left(a_{1}, b_{1}=1,2, \ldots, r\right) .
$$

Equation (4.6) now follows from the convergent series criterion.

Because $F_{X X}(\lambda)$ is a continuous bounded monotonic function of $\lambda,(4 \cdot 6)$ implies $(4 \cdot 7)$ following a theorem of Polya.

In the proof of Theorem $4 \cdot 3$ we will make use of the identity

$$
E\left(Y_{1} Y_{2} \ldots Y_{k}\right)=\sum_{\nu} \operatorname{cum}\left\{Y_{j}\left(j \in v_{1}\right)\right\} \ldots \operatorname{cum}\left\{Y_{j}\left(j \in \nu_{p}\right)\right\}
$$

where the summation is over all partitions $\left(\nu_{1}, \nu_{2}, \ldots, \nu_{p}\right)(p=1,2, \ldots, k)$ of the integers $1,2, \ldots, k$.

Proof of Theorem $4 \cdot 3$. We note that the various cumulant spectra of $\mathbf{X}(t)$ are bounded following Assumption I. If we note this and use (8.1I) above with Theorem $4 \cdot 3$, then for $n$ a positive integer

$$
\begin{aligned}
\mid T^{n} E\left(\left[I_{a b}^{(T)}\left(\alpha_{1}\right)-E\left\{I_{a b}^{(T)}\left(\alpha_{1}\right)\right\}\right]\right. & \left.\ldots\left[I_{a b}^{(T)}\left(\alpha_{2 n}\right)-E\left\{I_{a b}^{(T)}\left(\alpha_{2 n}\right)\right\}\right]\right) \mid \\
& \leqslant K \Sigma\left\{\frac{\left|\Delta^{(T)}\left(\alpha_{\nu_{1}} \pm \alpha_{\nu_{2}}\right)\right|^{2}}{T} \ldots \frac{\left|\Delta^{(T)}\left(\alpha_{\nu_{2 n-1}} \pm \alpha_{\nu_{2 n}}\right)\right|^{2}}{T}\right\}
\end{aligned}
$$

for some $K>0$, where the summation extends over all permutations $\left(v_{1}, v_{2}, \ldots, v_{2 n}\right)$ of $(1,2, \ldots, 2 n)$ and all choices of \pm .

Therefore,

$$
\begin{aligned}
T^{n} E\left(\mid\left[F_{a b}^{(T)}(\lambda)-E\right.\right. & \left.\left.\left\{F_{a b}^{(T)}(\lambda)\right\}\right]-\left.\left[F_{a b}^{(T)}(\mu)-E\left\{F_{a b}^{(T)}(\mu)\right\}\right]\right|^{2 n}\right) \\
& \leqslant K \Sigma \int_{\mu}^{\lambda} \ldots \int_{\mu}^{\lambda} \frac{\left|\Delta^{(T)}\left(\alpha_{\nu_{1}} \pm \alpha_{\nu_{2}}\right)\right|^{2}}{T} \cdots \frac{\left|\Delta^{(T)}\left(\alpha_{\nu_{2 n-1}} \pm \alpha_{\nu_{2 n}}\right)\right|^{2}}{T} d \alpha_{1} \ldots d \alpha_{2 n} \\
& \leqslant L|\lambda-\mu|^{n}
\end{aligned}
$$

for some $L>0$ as we may integrate out $n$ of the $\alpha^{\prime}$ 's to remove the $\Delta^{(T)}$ functions and then note that the remaining $\alpha$ 's range from $\mu$ to $\lambda$.

It follows from $(3 \cdot 1)$ that there exists $M>0$ such that

$$
\left|\left[E\left\{F_{a b}^{(T)}(\lambda)\right\}-F_{a b}^{(T)}(\lambda)\right]-\left[E\left\{F_{a b}^{(T)}(\mu)\right\}-F_{a b}^{(T)}(\mu)\right]\right| \leqslant M|\lambda-\mu| .
$$


The combination of $(8 \cdot 13)$ and $(8 \cdot 14)$ gives

$$
T^{n} E\left|\left\{F_{a b}^{(T)}(\lambda)-F_{a b}(\lambda)\right\}-\left\{F_{a b}^{(T)}(\mu)-F_{a b}(\mu)\right\}\right|^{2 n} \leqslant N|\lambda-\mu|^{n}
$$

for some $N>0$.

The indicated theorem now follows from the multivariate extension of the principal theorem of Lamperti (1962).

Proof of Theorem $5 \cdot 1$. We note that

$$
m_{a_{j} b_{j}}^{(T)}\left(u_{j}\right)=\int_{-\pi}^{\pi} I_{a_{j} b_{j}}^{(T)}\left(\alpha_{j}\right) \exp \left(i u \alpha_{j}\right) d \alpha_{j} \quad(j=1,2, \ldots, k),
$$

and so $(5 \cdot 4),(5 \cdot 5)$ and $(5 \cdot 6)$ follow directly by the arguments used in the proof of Theorem $4 \cdot 1$.

Proof of Corollary. We may write

$$
\begin{aligned}
m_{a_{j} b_{j}}^{(T)}\left(u_{j}\right) & =\int_{0}^{\pi} \exp (i u \alpha) d\left\{F_{a_{j} b_{j}}^{(T)}(\alpha)+F_{b_{j} a_{j}}^{(T)}(\alpha)\right\} \\
& =\left\{F_{a_{j} b_{j}}^{(T)}(\pi)+F_{b_{j} a_{j}}^{(T)}(\pi)\right\}-i u \int_{0}^{\pi}\left\{F_{a_{j} b_{j}}^{(T)}(\alpha)+F_{b_{j} a_{j}}^{(T)}(\alpha)\right\} \exp (i u \alpha) d \alpha
\end{aligned}
$$

if one integrates by parts. Equations $(5 \cdot 7)$ and $(5 \cdot 8)$ now follow from $(4 \cdot 6)$ and $(4 \cdot 7)$.

Proof of Theorem 5·2. This follows directly as did the proof of Theorem $4 \cdot 2$.

Proof of Theorem 5•3. Because the mapping of $\operatorname{Lip}_{\alpha}^{r \times r}(0, \pi)$ to $\mathscr{A}_{\alpha}^{r \times r}$ indicated by $(5 \cdot 10)$ is continuous, Theorem $5 \cdot 3$ follows directly from Theorem $5 \cdot 4$ once we note that

$$
T^{\frac{1}{2}}\left\{m_{a_{j} b_{j}}^{(T)}\left(u_{j}\right)-m_{a_{j} b_{j}}\left(u_{j}\right)\right\}=\int_{0}^{\pi} \exp (i u \alpha) d T^{\frac{1}{2}}\left\{F_{a_{j} b_{j}}^{(T)}(\alpha)-F_{a_{j} b_{j}}(\alpha)+F_{b_{j} a_{j}}^{(T)}(\alpha)-F_{b_{j} a_{j}}(\alpha)\right\}
$$

Proof of Theorem $6 \cdot 1$. Expression (6.3) follows directly from $(6 \cdot 2),(3 \cdot 1)$ and the definition of $H^{(T)}(\alpha)$. Turning to $(6 \cdot 4)$, from $(6 \cdot 2)$ we have

$$
\operatorname{cov}\left\{f_{a_{1} b_{1}}^{(T)}\left(\lambda_{1}\right), f_{a_{2} b_{2}}^{(T)}\left(\lambda_{2}\right)\right\}=\iint_{-\pi}^{\pi} H^{(T)}\left(\alpha_{1}\right) H^{(T)}\left(\alpha_{2}\right) \operatorname{cov}\left\{I_{a_{1} b_{1}}^{(T)}\left(\lambda_{1}-\alpha_{1}\right), I_{a_{2} b_{2}}^{(T)}\left(\lambda_{2}-\alpha_{2}\right)\right\} d \alpha_{1} d \alpha_{2}
$$

We will substitute into this expression from $(3 \cdot 2)$. Now in the case that $B_{T} T \rightarrow \infty$ we have

$$
\int_{-\pi}^{\pi} H^{(T)}(\alpha) T^{-1}\left|\Delta^{(T)}(\gamma-\alpha)\right|^{2} d \alpha=H^{(T)}(\gamma)+O\left(B_{T}^{-2} T^{-1}\right) \quad(-\pi \leqslant \gamma \leqslant \pi) .
$$

Also

$$
\int_{-\pi}^{\pi}\left|H^{(T)}(\alpha)\right|\left|\Delta^{(T)}(\gamma-\alpha)\right| d \alpha=O\left(B_{T}^{-1} \log T\right) .
$$

These two indicate that the covariance in question is given by

$$
\begin{aligned}
& T^{-1}\left\{\int_{-\pi}^{\pi} H^{(T)}(\alpha) H^{(T)}\left(\lambda_{2}-\lambda_{1}-\alpha\right) f_{a_{1} a_{2}}\left(\lambda_{1}-\alpha_{1}\right) f_{b_{1} b_{2}}\left(\alpha_{1}-\lambda_{1}\right) d \alpha\right. \\
& \left.\quad+\int_{-\pi}^{\pi} H^{(T)}(\alpha) H^{(T)}\left(\lambda_{2}+\lambda_{1}-\alpha\right) f_{a_{1} b_{2}}\left(\lambda_{1}-\alpha\right) f_{b_{1} a_{2}}\left(\alpha-\lambda_{1}\right) d \alpha\right\} \\
& \quad+2 \pi T^{-1} \iint_{-\pi}^{\pi} H^{(T)}\left(\alpha_{1}\right) H^{(T)}\left(\alpha_{2}\right) f_{a_{1} b_{1} a_{2} b_{2}}\left(\lambda_{1}-\alpha_{1}, \alpha_{1}-\lambda_{1}, \alpha_{2}-\lambda_{2}\right) d \alpha_{1} d \alpha_{2} \\
& \quad+O\left(B_{T}^{-2} T^{-2}\right)+O\left(B_{T}^{-1} T^{-3} \log T\right)
\end{aligned}
$$

from which $(6 \cdot 4)$ follows. 
Expression (6.5) follows by a similar, but cruder, argument using (3.4).

Proof of Theorem $6 \cdot 2$. We note that all cumulants of order greater than two tend to 0 as $T \rightarrow \infty$. This gives the result.

Proof of Theorem $6 \cdot 3$. This follows directly from Theorem $3 \cdot 2$.

I would like to thank Professor J. Durbin for a number of helpful comments.

\section{REFERENCES}

Anderson, T. W. (1958). Introduction to Multivariate Statistical Analysis. New York: Wiley.

ANDERSON, T. W. \& WALKER, A. M. (1964). On the asymptotic distribution of the autocorrelations of a sample from a linear stochastic process. Ann. Math. Statist. 35, 1296-1303.

BARTLETt, M. S. (1946). On the theoretical specification of sampling properties of auto-correlated time series. J. R. Statist. Soc. Suppl. 8, 27-41.

Bartlett, M. S. (1950). Periodogram analysis and continuous spectra. Biometrika 37, 1-16.

BartLett, M. S. (1966). An Introduction to Stochastic Processes, 2nd edition. Cambridge University Press.

Blackman, R. B. \& Tukey, J. W. (1958). The Measurement of Power Spectra. New York: Dover.

Brillinger, D. R. (1965). An introduction to polyspectra. Ann. Math. Statist. 36, 1351-74.

BRILLINGER, D. R. (1968). Estimation of the cross-spectrum of a stationary bivariate Gaussian process from its zeros. J. R. Statist. Soc. B 30, 145-59.

Brillinger, D. R. \& Rosenblatt, M. (1967). Asymptotic theory of estimates of $k$-th order spectra. In Advanced Seminar on Spectral Analysis of Time Series (ed. B. Harris), pp. 153-88. New York: Wiley.

Edwards, R. E. (1967). Fourier Series, vol. 1. New York: Holt, Rinehart and Winston.

GOodman, N. R. (1963). Statistical analysis based on a certain multivariate complex Gaussian distribution (an introduction). Ann. Math. Statist. 34, 152-77.

Grenander, U. \& Rosenblatt, M. (1957). Statistical Analysis of Stationary Time Series. New York: Wiley.

Hannan, E. J. (1960). Time Series Analysis. London: Methuen.

Hannan, E. J. (1967). The estimation of a lagged regression relation. Biometrika 54, 409-18.

IbraGtMov, I. A. (1963). On estimation of the spectral function of a stationary Gaussian process. Theory Probab. Appl. 8, 366-401.

Kawata, T. (1959). Some convergence theorems for stationary stochastic processes. Ann. Math. Statist. 30, 1192-214.

LAMPERTI, J. (1962). On convergence of stochastic processes. Trans. Amer. Math. Soc. 104, 430-5.

LeONov, V. P. \& Shiryaev, A. N. (1959). On a method of calculation of semi-invariants. Theory Probab. Appl. 4, 319-29.

LOMNICKI, Z. A. \& ZAREMBA, S. K. (1957). On some moments and distributions occurring in the theory of linear stochastic processes. Part I. Mh. Math. 61, 318-58.

Lomnicki, Z. A. \& ZAREMBA, S. K. (1959). On some moments and distributions occurring in the theory of linear stochastic processes. Part II. Mh. Math. 63, 128-68.

MALEVICH, T. L. (1964). The asymptotic behavior of an estimate for the spectral function of a stationary Gaussian process. Theory Probab. Appl. 9, 350-3.

MaLEVICH, T. L. (1965). Some properties of the estimators of the spectrum of a stationary process. Theory Probab. Appl. 10, 457-65.

Olshen, R. A. (1967). Asymptotic properties of the periodogram of a discrete stationary process. J. Appl. Prob. 4, 508-28.

Parzen, E. (1957a). A central limit theorem for multilinear stochastic processes. Ann. Math. Statist. $28,252-5$.

Parzen, E. (1957b). On consistent estimates of the spectrum of a stationary time series. Ann. Math. Statist. 28, 329-48.

Parzen, E. (1961). An approach to time series analysis. Ann. Math. Statist. 32, 951-89.

RAO, S. T. (1967). On the cross periodogram of a stationary Gaussian vector process. Ann. Math. Statist. 38, 593-7.

Rosenblatt, M. (1959). Statistical analysis of stochastic processes with stationary residuals. In Studies in Probability and Statistics (ed. U. Grenander), pp. 246-75. New York: Wiley. 
Rosenblatt, M. (1962). Asymptotic behavior of eigenvalues of Toeplitz forms. J. Math. Mech. 11, 941-50.

Slutsky, E. E. (1934). Alcune applicazioni dei coefficienti di Fourier all'analisi delle funzioni aleatorie stazionarie. Gion. Ist. Ital. Attuari 5, 1-50.

TUkey, J. W. (1967). An introduction to the calculations of numerical spectrum analysis. In Advanced Seminar on Spectral Analysis of Time Series (ed. B. Harris), pp. 25-46. New York: Wiley.

WaнвA, G. (1968). On the distribution of some statistics useful in the analysis of jointly stationary time series. Ann. Math. Statist. 39, 1849-62.

WALKER, A. M. (1954). The asymptotic distribution of serial correlation coefficients for autoregressive processes with dependent residuals. Proc. Camb. Phil. Soc. 50, 60-4.

WALKER, A. M. (1965). Some asymptotic results for the periodogram of a stationary time series. J. Aust. Math. Soc. 5, 107-28.

[Received December 1968. Revised February 1969] 